analysis, significant associations were found between new-onset sciatica and age and obesity. In adjusted analysis, significant associations were found for obesity and mental workload in a qualitative aspect after controlling for age and gender. Consequently, in multivariate analysis with all the potential risk factors, obesity remained statistically significant (OR: 1.80; 95\% CI: 1.19-2.71) while age $(\geq 50$ years vs. $<40$ years) was almost significant (OR: 1.55, 95\% CI: 0.99-2.44).

Conclusions In previously asymptomatic Japanese workers, the risk of developing new-onset sciatica is mediated by individual factors such as age and obesity. Our findings suggest that the management of obesity may prevent new-onset sciatica.

\section{DETERMINANTS OF BACK-PAIN IN THE EUROPEAN COUNTRIES. A CROSS-SECTIONAL ANALYSIS OF DATA FROM THE FIFTH EUROPEAN WORKING CONDITIONS SURVEY}

${ }^{1}$ A F Farioli, ${ }^{1}$ Quaglieri, ${ }^{1}$ Curti, ${ }^{1}$ Mattioli, ${ }^{1}$ Violante, ${ }^{2}$ Palmer, ${ }^{2}$ Coggon. ${ }^{1}$ University of Bologna, Bologna, Italy; ${ }^{2}$ MRC Lifecourse Epidemiology Unit, University of Southampton, Southampton, United Kingdom

\subsection{6/oemed-2013-101717.337}

Objectives Prevalence of back-pain in European countries has been reported to vary considerably. We used data from the fifth European Working Conditions Survey (EWCS) to explore the role of personal, occupational, and country socioeconomic characteristics in determining the national prevalence of back-pain.

Methods The EWCS was conducted in 34 countries during 2010. 43,816 subjects were interviewed using a standardised questionnaire. We analysed the prevalence of back-pain over the last year. Occupational and personal exposures investigated include: age, gender, biomechanical risk factors (carrying or moving loads; lifting or moving people; standing; tiring or painful positions; vibrations); job-demand control model; educational level; socio-economic status; housework or gardening; caring for children or elderly/disabled; performing housework or gardening; doing sporting, cultural or leisure activity outside home; somatisation tendency. We also studied the role of country socioeconomic characteristics. Analyses were performed with logistic or Poisson regression models incorporating sampling weights and with standard error clustered on NUTS regions. Multilevel models were fitted to study country socioeconomic characteristics.

Results 30,066 workers entered the main analysis. With the exception of gender, all personal and occupational characteristics were associated with back-pain in multivariate analysis; somatising tendency was the strongest predictor of the symptoms. Country socioeconomic characteristics were associated with back-pain in univariate analysis. However, no association was found after adjustment by subject-level characteristics. National prevalence of back-pain ranged between 22.1\% (Ireland) and $64.2 \%$ (Portugal). Neighbouring countries frequently had similar prevalences. Adjustment for personal and occupational risk factors did not explain all of the variation in prevalence of backpain among European countries.

Conclusions The high variability in prevalence of back-pain among European countries was not explained by subject-level characteristics. The inclusion of current country socioeconomic characteristics did not improve the fit of multivariate statistical models. Group-level characteristics and cultural factors should be investigated.
338 THE RISK OF MUSCULOSKELETAL DISORDERS IN A COHORT OF DANISH BAGGAGE HANDLERS

${ }^{1} \mathrm{~S}$ H B Bern Hvid, 'Brauer, ${ }^{2}$ Møller Lauenborg, ${ }^{3}$ Koblauch, ${ }^{2}$ Thygesen, ${ }^{1}$ Thomsen, ${ }^{3}$ Simonsen, ${ }^{3}$ Alkjær, ${ }^{2}$ Helweg-Larsen, ${ }^{1}$ Mikkelsen. ${ }^{1}$ Bispebjerg University Hospital, Copenhagen, Denmark; 'University of Southern Denmark, Copenhagen, Denmark; ${ }^{3}$ Department of Neuroscience and Pharmacology, University of Copenhagen, Copenhagen, Denmark

\subsection{6/oemed-2013-101717.338}

Objectives To investigate associations between heavy lifting and musculo-skeletal disorders in a group of baggage handlers. It is generally accepted that heavy lifting and lifting in kneeling and stooped positions are risk factors for musculo-skeletal disorders in the shoulders, knees and lower back region. However, the influence of magnitude and time of heavy lifting on the risk of musculoskeletal disorders is still unclear. Baggage handlers are daily exposed to heavy lifting in stooped, squatting or kneeling positions in constrained spaces and hence they are a good study group for our objective. This abstract report the first results based on questionnaire data and will be supplemented later with objective data on lifting loads and diagnosed musculoskeletal disorders.

Methods We established a cohort of baggage handlers employed at Copenhagen Airport during the period 1983 to 2012 ( $\mathrm{n}=$ 3093) and a reference cohort of men who worked in other unskilled occupations during the same period $(\mathrm{n}=2478)$. Data regarding work history, lifestyle and musculoskeletal complaints were collected using a self-administered questionnaire. The response rate was $70.5 \%$. Data were analysed using logistic regression.

Results The risk of self-reported pain in the shoulders, knees and lower back was significantly higher in the group of baggage handlers than in the reference group. The difference between the groups disappeared after adjusting for length of employment. Furthermore, the risk of pain increased with length of employment. For every 10 years of employment the risk of shoulder pain increased by $34 \%(\mathrm{OR}=1.34$, CI: $1.19-1.51)$, the risk of knee pain increased by $45 \%(\mathrm{OR}=1.45, \mathrm{CI}: 1.29-1.63)$ and the risk of lower back pain increased by $46 \%(\mathrm{OR}=1.46, \mathrm{CI}$ : 1.31-1.63). Adjusting for age, height and weight did not change the results.

Conclusion The risk of self-reported shoulder, knee and lower back pain increased with the length of employment as baggage handler.

\section{LIFE-TIME CUMULATIVE LIFT LOAD AND LUMBAR DISC DEGENERATION}

${ }^{1} \mathrm{~L}$ Guo, ${ }^{2}$ Hung, ${ }^{3}$ Shih, ${ }^{3}$ Chen, ${ }^{4}$ Hwang. ${ }^{1}$ National Taiwan University, Taipei, Taiwan; ${ }^{2}$ Chung Hwa University of Medical Technology/Biological Science and Technology, Tainan, Taiwan; ${ }^{3}$ National Taiwan University College of Medicine/Medical Imaging and Radiology, Taipei, Taiwan; ${ }^{4}$ National Taiwan University/Occupational Medicine and Industrial Hygiene, Taipei, Taiwan

\subsection{6/oemed-2013-101717.339}

Objectives Lumbar disc degeneration (LDD) has been associated with cumulative lift load. However, dose-response relationship has not been established in Asia workers. The goal of this study is to investigate whether a dose-response relationship exists between LDD and lifetime cumulative lift load on lumbar disc. Methods A total of 553 subjects who have ever worked were recruited. The participants received assessment with a questionnaire and magnetic resonance imaging (MRI) of the L-S spine. 